\title{
Long-term postoperative survival of a gastric cancer patient with numerous para-aortic lymph node metastases
}

\author{
Takao Inada, Yoshiro Ogata, Iwao Ozawa, Moriaki Tomikawa, Seichiro Yamamoto, Jiro Ando, \\ Shoichi Hishinuma, Hideaki Shimizu, and Kenjiro Kotake \\ Department of Surgery, Tochigi Cancer Center, 4-9-13 Yohnan, Utsunomiya 320-0834, Japan
}

\begin{abstract}
:
A 56-year-old man with advanced gastric cancer was referred to our hospital. Preoperative abdominal computed tomography revealed numerous enlarged lymph nodes, including the lymph nodes of the paraaortic region. The patient underwent total gastralectomy, splenectomy, left-adrenalectomy and resection of the body and tail of the pancreas by Appleby's method, along with paraaortic lymph node dissection. Microscopic examination revealed that the tumor was a solid type, poorly differentiated adenocarcinoma, which displayed invasion of the serosal surface. There were apparently many lymph node metastases. We identified 31 cancer-positive paraaortic nodes, while the total number of lymph node metastases was 81. It was not possible to administer sufficient postoperative adjuvant chemotherapy, as the patient experienced postoperative complications, including pancreatic fistula and watery diarrhea. Despite the lack of sufficient chemotherapy, the patient has subsequently remained disease-free for 9 years and 3 months, and continues to visit our hospital as an outpatient. In conclusion, we wish to emphasize the need for a critical application of paraaortic lymph node dissection as one modality of multidisciplinary treatment in patients with advanced gastric cancer in whom paraaortic lymph node metastasis is strongly suspected preoperatively.
\end{abstract}

Key words: gastric cancer, para-aortic lymph node dissection

\section{Introduction}

D2 resection, in which group 1 and 2 lymph nodes are dissected in patients with gastric cancer, is the standard method used for lymphadenectomy in Japan, and it is commonly performed in most institutions that deal with gastric cancer patients [1]. Extensive radical lymphadenectomy, in which the dissection is performed up to the paraaortic lymph nodes, is also commonly

Offprint requests to: $\mathrm{T}$. Inada

Received on July 7, 1999; accepted on Oct. 12, 1999 practiced [2-4]. Prophylactic paraaortic lymphadenectomy is typically performed in patients in whom the cancer is advanced; for example, when there is serosal invasion, or when there is macroscopically identified group 2 lymph node metastasis. We have been performing paraaortic lymph node dissection at our Institute for approximately 10 years [5].

Improvements in diagnostic procedures, such as computed tomography, have made it possible to detect the characteristic enlargement of the paraaortic lymph nodes preoperatively. Note, however, that there is some controversy surrounding the practice of performing curative resection in patients with advanced cancer in whom enlarged paraaortic lymph nodes are detected preoperatively and/or intraoperatively.

We report a patient with advanced gastric cancer, in whom we identified 31 metastatic paraaortic lymph nodes, who has survived for more than 5 years without a recurrence of the cancer.

\section{Case report}

The patient, a 56-year-old man, presented in complaining of dysphasia that had been occurring since December 1989. He visited a nearby surgical hospital and gastrointestinal radiography revealed a gastric cancer in the cardiac portion. He was referred to our hospital in February 1990 for treatment and admitted on February 28.

\section{Preoperative examination}

On admission to the hospital, the patient was $168.7 \mathrm{~cm}$ tall and weighed $69.5 \mathrm{~kg}$. His general condition was good and we could not identify any physical abnormalities. Blood tests revealed slight anemia, with a hemoglobin value of $10.9 \mathrm{~g} / \mathrm{dl}$. Results of other assays, including those for tumor markers, were all within the normal range. 
Gastrointestinal radiography revealed a giant type 3 advanced gastric cancer extending from the esophagogastric junction to the angle of the stomach (Fig. 1). Endoscopic examination confirmed the diagnosis of type 3 gastric cancer and indicated that the tumor had invaded the esophagus about 1 or $2 \mathrm{~cm}$ beyond the esophagogastric junction. Biopsy specimens obtained endoscopically identified poorly differentiated adenocarcinoma.

Abdominal computed tomography revealed enlargement of numerous lymph nodes, including the bilateral large paraaortic lymph nodes (Fig. 2). Using the

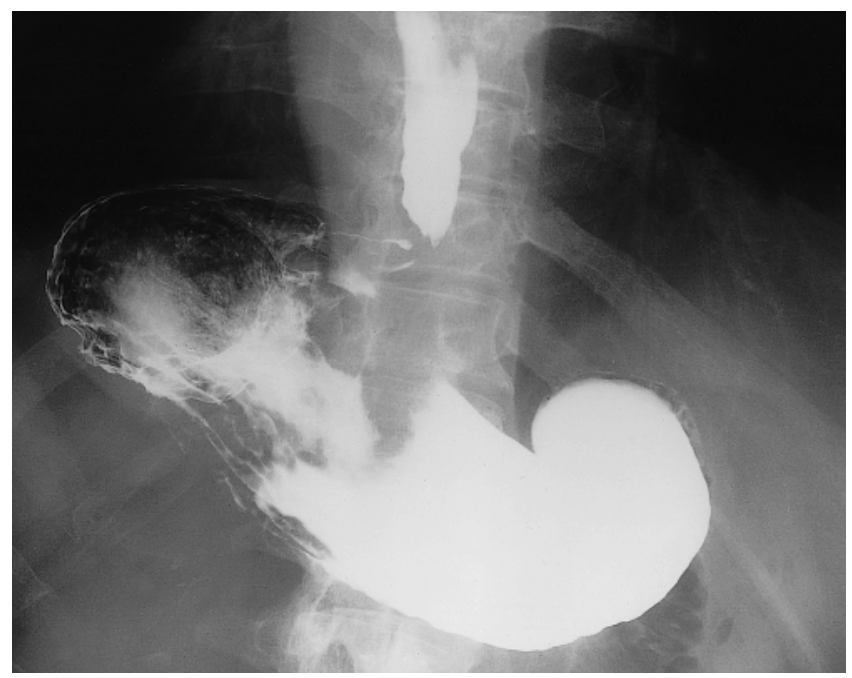

Fig. 1. Radiological examination shows defect and wall irregularity of the lesser curvature from the esophagogastric junction to the middle body (prone position)
Japanese classification of gastric carcinoma [6], we made a positive preoperative diagnosis of lymph node metastasis in the following regions: 1, 3, 7, 8a, 9, 10, 11, $14 \mathrm{a}, 16 \mathrm{a}_{2}$, and $16 \mathrm{~b}_{1}$. We could not identify any hepatic or peritoneal involvement; however, there was evidence of slight pleural effusion bilaterally in the chest. With the exception of the lymph nodes, there was no evidence of any additional distant metastasis and it was decided that the patient should receive a gastrectomy.

\section{Surgical findings}

A laparotomy was performed on March 12, via an upper and lower midline incision. The main intraoperative findings included the discovery that the gastric cancer had invaded as far as the middle portion of the pancreas (T4), and lymph node metastases were found to extend as far as the paraaortic region (N4). Hepatic and peritoneal involvement was apparently absent $(\mathrm{H} 0, \mathrm{P} 0)$. Lavage cytology obtained from the pelvic cavity and touch-smear cytology of the gastric serosal surface were cancer-negative (CY0). We therefore proceeded with a macroscopically curative resection, to which we added a left eighth intercostal thoracotomy. As the cytology of the left pleural effusion was also negative, we performed total gastralectomy, splenectomy, leftadrenalectomy, and resection of the body and tail of the pancreas by Appleby's method. A D4 lymph node dissection was performed, as well as a paraaortic lymph node dissection from the level of the aortic hiatus to the level of the aortic bifurcation, and a macroscopically curative resection was performed (Fig. 3). Reconstruction was achieved by esophagojejunal anastomosis in a Roux-en-Y fashion.
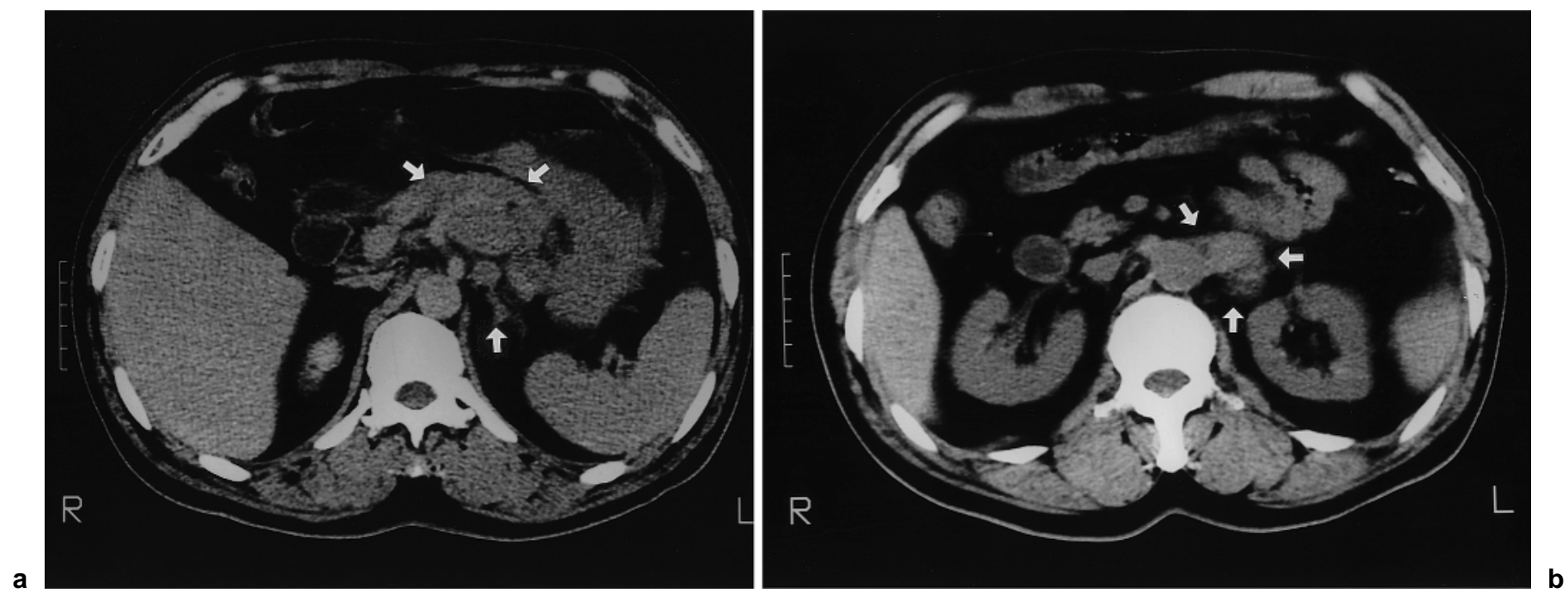

Fig. 2a,b. Abdominal computed tomography: enlarged lymph nodes (arrows) around the abdominal aorta are seen a from the level of the superior mesenteric artery to $\mathbf{b}$ the level of the renal hilus 


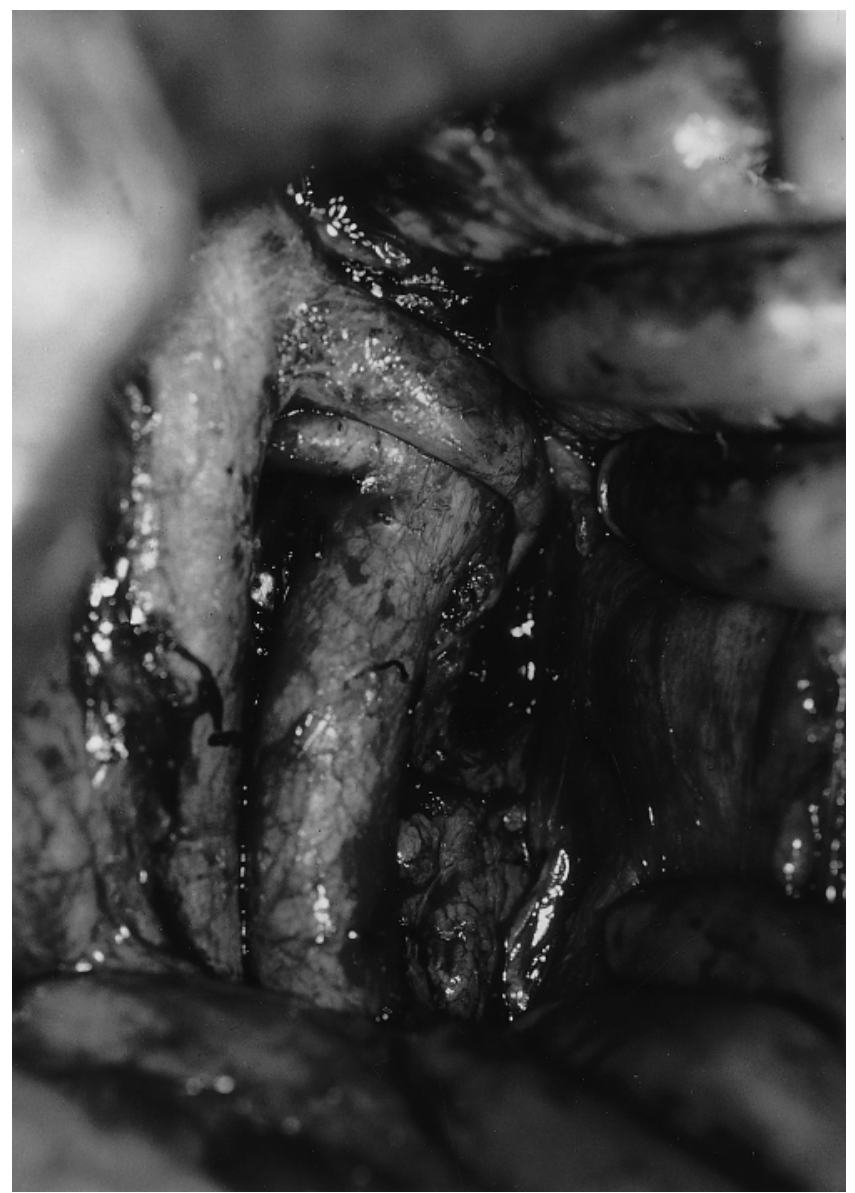

Fig. 3. Intraoperative view of the patient after resection of the paraaortic lymph nodes

\section{Pathological findings}

The resected specimen was a type 3 advanced gastric cancer, measuring $10 \times 10 \mathrm{~cm}$ and located near the posterior wall of the fundus (Fig. 4). Histopathologically, large tumor cells with atypical nuclei showed medullary growth and few glandular structures. Slight infiltration of lymphoid and plasma cells was observed (Fig. 5). Histological analysis confirmed that the tumor cell was a solid-type poorly differentiated adenocarcinoma with marked lymphatic and moderate venous invasion. Tumor invasion was observed on the serosal surface (por1, ly3, v2, se). A total of 110 lymph nodes were resected and metastasis was identified in 81 of these, determined using the Japanese classification of gastric carcinoma [6]. The cancer-positive lymph nodes were in the following regions: $3,7,8 \mathrm{a}, 8 \mathrm{p}, 9,10,11,14 \mathrm{a}$, and 16 . We also identified 31 paraaortic lymph node metastases, located in the following regions: $16 \mathrm{a} 2$ int, ant $(8 / 8), 16 \mathrm{a} 2$ lat (7/7), 16b1 int, ant (10/12), 16b1 lat (6/10), and 16b2 ant $(0 / 3)$.

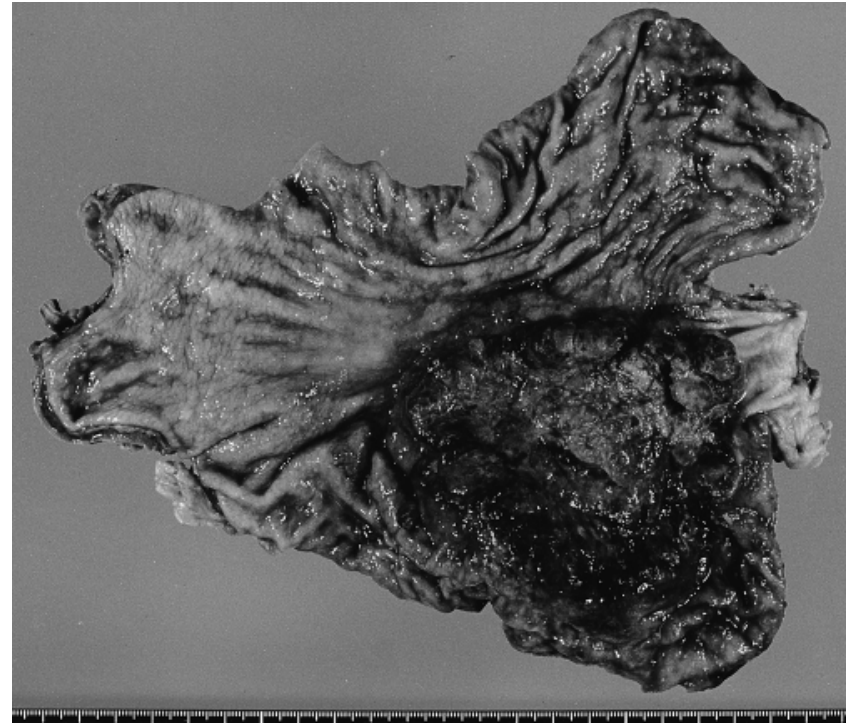

Fig. 4. Resected specimen of the stomach shows a type 3 gastric cancer measuring $10 \times 10 \mathrm{~cm}$

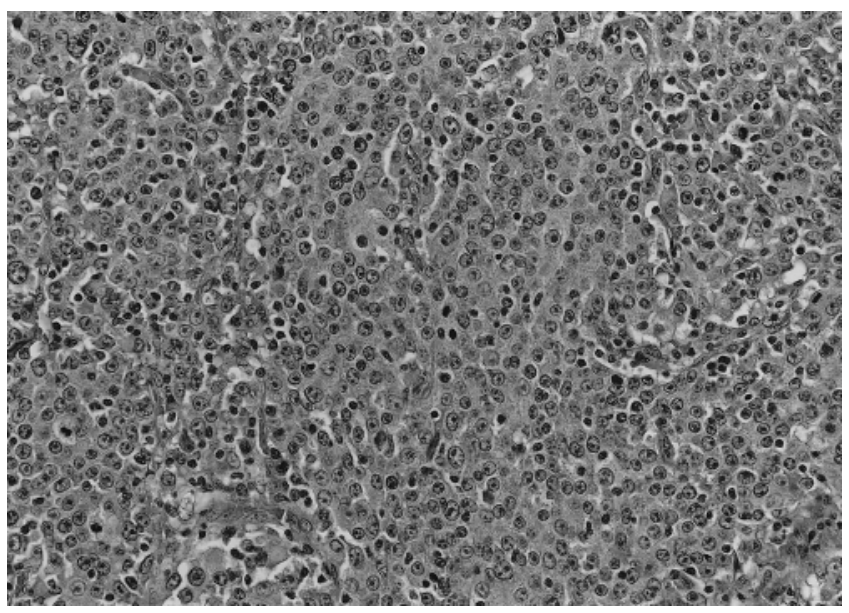

Fig. 5. High-power view of solid-type poorly differentiated adenocarcinoma with slight infiltration of lymphoid and plasma cells. $\mathrm{H} \& \mathrm{E}, \times 260$

\section{Postoperative course}

Adjuvant chemotherapy was performed with an intravenous infusion of mitomycin $C(20 \mathrm{mg} /$ body immediately after the operation and $10 \mathrm{mg} / \mathrm{body}$ during the following day). We also administered futrafur suppositories daily $(750 \mathrm{mg} /$ body per day) commencing the day after the operation. On the eighth postoperative day, however, we observed a purulent discharge from the intraabdominal drainage tube, which prompted us to terminate the futrafur treatment. Pan- 
creatic fistula was recognized on the tenth postoperative day.

The pancreatic fistula resolved after approximately 1 month with conservative treatment and the patient was discharged from the hospital on the 79th postoperative day. His weight at the time of discharge was $49 \mathrm{~kg}$, representing a total weight loss during hospitalization of approximately $20 \mathrm{~kg}$. After he was discharged, adjuvant chemotherapy could not be performed because of persistent watery diarrhea, which lasted for approximately 3 years, and required treatment with opium tincture. The patient has subsequently remained healthy and free of any signs of cancer recurrence as of June 1999.

\section{Discussion}

We report a patient who has survived for more than 9 years after surgical treatment for advanced gastric cancer. The cancer displayed serosal invasion; however, there was no macroscopic involvement of the liver and peritoneum. The patient received an extensive lymphadenectomy, including paraaortic lymph node dissection, and macroscopically curative resection was performed. We have documented 26 cases involving macroscopically curative operations for the treatment of gastric cancer with microscopically positive paraaortic lymph node metastasis within the same 10-year period (1990-1999). The 5-year survival rate of these patients was $25.1 \%$, and six patients (including the present patient) have survived for more than 5 years without a recurrence of cancer [7].

A nationwide questionnaire of 53 Japanese patients who have survived for more than 5 years after extensive resection of positive paraaortic lymph nodes revealed the following clinicopathological features for long-term survival: the number of positive paraaortic nodes was less than 3 , the growth type was expansive, the size of the gastric cancer tended to be relatively small, and there was never any liver metastasis or peritoneal dissemination [8]. Apart from the present patient, in the other five patients we have observed who have survived for more than 5 years, the number of cancer-positive paraaortic lymph nodes was two or fewer. Note that 31 positive paraaortic lymph nodes were found in the present patient, and the total number of lymph node metastases was 81 . It would seem, therefore, that the long-term survival of our patient represents a somewhat rare occurrence.

As was found in our patient reported here, some patients with gastric cancer that is associated with many lymph node metastases have been successfully treated with a gastrectomy in conjunction with lymph node dissection. Among those patients who had a recurrence of cancer, most commonly the cancer was found in the peritoneum [9].

Among the 26 patients with paraaortic lymph node metastasis that we have treated, the patients who displayed positive peritoneal lavage cytology all subsequently died within 2 years after a recurrence in the peritoneum. Conversely, we have found that the 5-year survival rate in the patients in whom the peritoneal lavage cytology was negative $(n=17)$ is unexpectedly high, at $33.5 \%$. At present, therefore, we make the decision to pursue paraaortic lymph node dissection on the basis of the results of the peritoneal lavage cytology.

In the present patient, the peritoneal lavage cytology, the touch smear of the gastric serosa, and the left pleural effusion were all cancer-negative. We therefore pursued an extended radical operation, employing Appleby's method, in conjunction with a dissection of the enlarged paraaortic lymph nodes. Fortunately, the treatment yielded long-term survival and the absence of any recurrence of the disease. However, the patient's general condition after the surgery was poor, with marked weight loss and persistent watery diarrhea being the principal sequellae. We therefore stress that the greatest care must be taken in making the decision to perform a dissection of enlarged paraaortic lymph nodes. Massive lymphorrhea frequently occurs after paraaortic lymph node dissection because of disruption of the retroperitoneal lymphatic vessels, and the possibility that cancer cells may scatter into the abdominal cavity via the lymphatic vessels cannot be excluded. For these reasons, we propose that adjuvant therapy is necessary under these circumstances. In our patient, intensive adjuvant chemotherapy could not be performed because of the postoperative complications; however, the possibility that the insufficient chemotherapy contributed to the patients favorable prognosis is undeniable.

Recently, favorable results have been reported with the preoperative administration of chemotherapy. Yonemura et al. [10] reported that the postoperative survival rate of patients with high-grade advanced gastric cancer was significantly improved by neoadjuvant chemotherapy. We now administer neoadjuvant chemotherapy for those patients in whom paraaortic lymph node metastasis has been positively diagnosed preoperatively.

In conclusion, in the patient reported here, macroscopic curative resection with the dissection of paraaortic lymph nodes appearred to promote long-term survival. Therefore, we recommend that this procedure be included in the multidisciplinary treatment of patients with advanced gastric cancer, including those patients in whom a positive diagnosis of lymph node metastasis is made preoperatively. 


\section{References}

1. Maruyama K, Gunven P, Okabayashi K, Sasako M, Kinoshita T. Lymph node metastases of gastric cancer. General pattern in 1931 patients. Ann Surg 1989;210:596-602.

2. Maeta M, Saito H, Kondo A, Yamashiro H, Tsujitani S, Ikeguchi $\mathrm{M}$, et al. Effects of super-extended para aortic lymphadenectomy (PAL) on biological responses in totally gastrectomized patients with T3 or T4 gastric cancer. Gastric Cancer 1998;1:57-63.

3. Yonemura Y, Katayama K, Kamata T, Fushida S, Segawa M, Ooyama $\mathrm{S}$, et al. Surgical treatment of advanced gastric cancer with metastasis in paraaortic lymph nodes. Int Surg 1991;76:2225.

4. Takahashi S, Takenaka A, Tokuda H. Evaluation of D4 dissection for gastric cancer. In: Nishi M, Sugano H, Takahashi T, editors. First International Gastric Cancer Congress. Bologna: Monduzzi Editore, 1995:1147-50.

5. Inada T, Ogata Y, Andoh J, Ozawa I, Matsui J, Hishinuma S, et al. Significance of para-aortic lymph node dissection in patients with advanced and recurrent gastric cancer. Anticancer Res 1994;14:677-82.

6. Japanese Gastric Cancer Association. Japanese classification of gastric carcinoma. 2nd English Ed. Gastric Cancer 1998;1:1024.

7. Inada $\mathrm{T}$, Ogata $\mathrm{Y}$, Kikuyama S. Indication and prognosis of paraaortic lymph node dissection of gastric cancer. In: Kim JK, Min JS, Mok YJ, editors. Third International Gastric Cancer Congress. Bologna: Monduzzi Editore, 1999:525-30.

8. Aiko T, Saihara T, Hokita S, Ishigami J, Natsugoe S, Takao S. Rational lymphadenectomy for gastric cancer. Is it necessary to limit or extend dissection (in Japanese)? Jpn J Gastroenterol Surg 1994;27:968-73.

9. Katai H, Maruyama K, Sasako M, Sano T, Okajima K, Kinoshita $\mathrm{T}$, et al. Mode of recurrence after gastric cancer surgery. Dig Surg 1994;11:99-103.

10. Yonemura Y, Sawa T, Kinoshita K, Matsuki N, Fushida S, Tanaka S, et al. Neoadjuvant chemotherapy for high-grade advanced gastric cancer. World J Surg 1993;17:256-62. 